\title{
The molecular origin of stress generation in worm-like micelles, using a rheo-SANS LAOS approach
}

\author{
S. Rogers, ${ }^{a}$ J. Kohlbrecher ${ }^{b}$ and M. P. Lettinga $* a$ \\ Received 12th March 2012, Accepted 31st May 2012 \\ DOI: $10.1039 / \mathrm{c} 2 \mathrm{sm} 25569 \mathrm{c}$
}

We combine large-amplitude oscillatory shear (LAOS) and time-resolved small angle neutron scattering (t-SANS) measurements on the dynamic response of a dispersion of surfactant worm-like micelles. By viewing the response as being the result of a sequence of physical processes, we observe new features of the system response. We identify three distinct frequency regimes including a high frequency Maxwell-like, a yielding, and a settled flow regime. For each regime we identify the molecular origins, on a Kuhn segment scale, of the stress response. We show the ability of the material to 'over-orient' pre-yielding, compared with stationary flow. Finally, we present a stress-orientation rule which is rate-based at low frequencies and strain-based at high frequencies.

\section{Introduction}

Flow instabilities have been the focus of fundamental and applied research because of the ease with which complexity can be induced and studied, and the implications that such instabilities have on industrial processing. ${ }^{1}$ At low Reynolds numbers, flow instabilities are related to the shear-thinning and thickening behavior of the fluid. ${ }^{2,3}$ Surfactant worm-like micellar (WLM) solutions are a particularly interesting class of material that show such behavior and cetylpyridinium chloride-sodium salicylate (CPyCl-NaSal) is a well-studied example. ${ }^{4}$ WLM solutions are often referred to as 'living' systems because they display a combination of reptation dynamics of polymeric systems, and scission and recombination. ${ }^{5}$ Flow instabilities in $\mathrm{CPyCl}-\mathrm{NaSal}$ solutions are manifested in the formation of shear bands with low ordering and shear rate coexisting with shear bands with high ordering and shear rate layered in the gradient direction. ${ }^{6-9}$ These 'gradient bands' can then evolve into structure formation in the vorticity direction. ${ }^{10-12}$

Though there is a detailed level of understanding concerning the mechanism and kinetics of SB formation, ${ }^{2-4,13}$ there is a corresponding lack of knowledge of the molecular state of the material prior to this. The amount of deformation the material can withstand and the degree of alignment it exhibits before and after the onset of flow instabilities are unclear. In this work we seek to investigate these states by monitoring the transient macro- and micro-structural responses of a $6 \% \mathrm{CPyCl}-\mathrm{NaSal}$ dispersion to deformation rates under which stable flow cannot be sustained.

${ }^{a}$ ICS-3, Institut Weiche Materie, Forschungszentrum Jülich, D-52425 Jülich, Germany. E-mail: p.lettinga@fz-juelich.de

${ }^{b}$ Laboratory for Neutron Scattering, Paul Scherrer Institut, 5232 Villigen PSI, Switzerland
In the case of WLMs information regarding the macromicroscopic link is contained in the stress-orientation relationship. This general relationship describes a functional dependence of one parameter on the other. These two are often obtained via an intermediary parameter, $x$, whereby derivatives of the orientation and stress tensors with respect to $x$ are combined into a single relationship which is independent of $x$. Many forms of stress-orientation relationships have been identified under steady shear conditions, where shear rate plays the role of the intermediary. ${ }^{14-16}$

To elucidate the stress-orientation relationship before and after the onset of flow instabilities, an experimental method is required that probes the orientation in the sample on the relevant time scales: the equilibrium relaxation which accounts for the combination of reptation and scission dynamics and the timescale of shear band formation ${ }^{7,17-20}$ and migration. ${ }^{21}$

There are various examples where orientation of a sheared sample is determined. In the steady-state, steady-shear limit, where instabilities have sufficient time to form, orientation has been measured by in situ Small Angle Neutron Scattering (SANS), ${ }^{22-27}$ NMR,,${ }^{8,28,29}$ and birefringence. ${ }^{27,30,31}$ These experiments supplied not only information about the degree by which Kuhn segments are ordered, but also provided evidence for the existence of shear bands. A qualitative correspondence between the orientational ordering of Kuhn segments and macroscopic rheological stress has been found under steady-shear conditions by linking rheo-SANS measurements to the Giesekus model. ${ }^{24}$ Most of these experimental methods lack the time-resolution required to access the desired information. As of publication, the only technique with sufficient time resolution to follow the ordering for such fast kinetics is measurement of birefringence, which has been employed to follow the kinetics of SB formation, using transient steady-shear step-rate methodologies. ${ }^{7,17-19,32}$ 
We present here large-amplitude oscillatory shear (LAOS) rheological measurements, performed in parallel with timeresolved small angle neutron scattering (t-SANS) ${ }^{33,34}$ on a $6 \mathrm{wt}^{\mathrm{t}} \%$ $\mathrm{CPyCl}-\mathrm{NaSal}$ micellar dispersion. With this approach, we simultaneously access the evolution of the Kuhn segment alignment and the macroscopic stress under oscillatory shear. ${ }^{35} \mathrm{We}$ choose t-SANS as an experimental technique because with SANS we can very specifically select the length scale at which the orientational ordering is probed. The length scale selected is that of the Kuhn segments. A stroboscopic collection of neutrons is employed to obtain the time-resolution needed to observe the transient phenomena. Using LAOS, we probe material states that are inaccessible to steady-shear step-rate methodologies $^{7,17-20}$ that have been used in the past to study transience. By altering the frequency at which strain is applied, we change the time taken to reach the maximum shear rate. These experiments should thus be thought of as sweeping up and down the flow curve at various speeds, passing through low shear-rate stable flow conditions every half-period. In this way we control the viscoelastic states that we probe, which range from well-studied flowing banded states ${ }^{6,7,17,18,20}$ at low frequencies and high shear rates, to previously inaccessible highly aligned elastic states at intermediate and high frequencies.

When analyzing the orientation and stress responses to LAOS, we do not limit ourselves to linear-algebraic analysis techniques, ${ }^{36,37}$ but instead recognize that the response of the system can represent a sequence of physical processes. ${ }^{38}$ By doing so, we are able to define time windows within which clear relationships between the stress and orientational responses can be identified.

The layout of this paper is as follows: we first explain the methodology used to analyze and interpret the data. Next, in the Experimental section, we show how t-SANS is used to obtain time-resolved information on the orientation of the Kuhn segments of the WLMs. We then present the results from experiments carried out under low shear rate amplitudes where responses are predominantly linear. The next section presents results from large (strain and shear rate) amplitude oscillations, and it is divided into three parts. We first discuss low frequency results where SBs have sufficient time to form, and compare our results with earlier steady-shear findings. Then we present results of the high frequency regime, where the responses are predominantly Maxwellian, with the exception of the observation of strain softening. We conclude the presentation of our results with an intermediate frequency regime in which yielding behavior in a shear banded state is observed. From the combination of these sections we deduce the microstructural information we set out to determine.

We summarize our findings in two parts showing the different regimes of responses we have identified in a Pipkin diagram and concatenating the stress-optical relationships obtained from each (bounded) frequency regime to form a general relationship that spans the entire three decade frequency window investigated.

\section{Methodology: data analysis}

We define the global equilibrium relaxation time $\lambda$ as being the inverse of the frequency where $G^{\prime}=G^{\prime \prime}$ in linear regime oscillatory shear tests. Under steady-shear conditions, a plateau is observed in the flow-curve at shear rates in excess of $1 / \lambda^{4,6-8} \lambda$ therefore sets the natural timescale for shear rate and angular frequency. The Weissenberg number (amplitude), $\mathrm{Wi}_{0}=\lambda \dot{\gamma}_{0}$, is the normalized shear rate (amplitude) and the Deborah number, $\mathrm{De}=\lambda \omega$, is the normalized angular frequency. The response of the WLMs is probed by selecting rate amplitudes, $\mathrm{Wi}_{0}$, of the oscillations well above $\left(\mathrm{Wi}_{0} \gg 1\right)$ and below $\left(\mathrm{Wi}_{0}<1\right)$ the onset shear rate of the stress plateau. For a fixed value of $\mathrm{Wi}_{0}$ strain is applied at frequencies that range from De $\gg 1$, where the system displays an elastic response, to frequencies whose period is much longer than the time needed for shear band formation. Previous experiments ${ }^{7,17,18,20}$ show that at high shear rates, this time is on the order of a few seconds. The period of the lowest frequency applied in this study is approximately two orders of magnitude longer. This experimental protocol therefore provides coverage of the Pipkin space spanned by $\mathrm{Wi}_{0}$ and De.

Because of the difficulties associated with interpretation of high-order harmonics of both Fourier and Chebyshev analyses $^{37,39}$ as recently discussed, ${ }^{36}$ we adopt a similar analysis methods to that used by Rogers et al. ${ }^{38}$ We choose to record shape-specific quantities based on the strain acquired since the previous reversal as well as the instantaneous rate at which specific events occur in oscillatory experiments. One specific event that is well defined is the point of maximum stress, or the point of maximum ordering. Unlike $G^{\prime}$ and $G^{\prime \prime}$, these points are well defined for all response shapes and lend themselves to physical interpretation easily. To provide some insight into how this can be used to determine viscoelastic properties, we determine here the position of the stress maximum in the linear viscoelastic Maxwell model. The stress response, $\sigma(t)$, of the Maxwell model to oscillatory shearing of strain amplitude $\gamma_{0}=$ $\mathrm{Wi}_{0} / \mathrm{De}$ and angular frequency $\omega=\mathrm{De} / \lambda$ is $\sigma(t)=\mathrm{Wi}_{0} / \mathrm{De}$ $\left[G^{\prime} \sin (t \mathrm{De} / \lambda)+G^{\prime \prime} \cos (t \mathrm{De} / \lambda)\right]$, where $G^{\prime}=G_{0} \frac{\mathrm{De}^{2}}{1+\mathrm{De}^{2}}$ is the storage modulus and $G^{\prime \prime}=G_{0} \frac{\mathrm{De}}{1+\mathrm{De}^{2}}$ is the loss modulus. $G_{0}$ is the elastic plateau modulus and $\lambda$ is the relaxation time. The maximum stress response of the Maxwell model is achieved when $t=\frac{\lambda \tan ^{-1}(\mathrm{De})}{\mathrm{De}}$ so that the amount of strain acquired since the previous reversal at the stress maximum is equal to $\gamma_{\text {acquired }} @ \sigma_{\max }=\mathrm{Wi}_{0} / \mathrm{De}+\mathrm{Wi}_{0} / \mathrm{De} \sin \left(\tan ^{-1}(\mathrm{De})\right)$. The instantaneous shear rate at the point of maximum stress is therefore equal to $\dot{\gamma} @ \sigma_{\max }=\dot{\gamma}_{0} \cos \left(\tan ^{-1} \mathrm{De}\right)$. The Maxwell model behaves liquid-like at frequencies much smaller than the inverse of the relaxation time, i.e. when De $\ll 1$. In this limit, the stress maximum occurs when strain equal to $\gamma_{0}$ has been acquired since the previous reversal and the instantaneous shear rate is $\dot{\gamma}_{0}$. When De $\gg 1$, an elastic response is elicited from the Maxwell model. This is reflected by the stress maximum occurring after a strain of $2 \gamma_{0}$ has been acquired since the previous reversal, which occurs when the shear rate is instantaneously zero. In the intermediate frequency regime, where $\mathrm{De} \approx 1$, a viscoelastic response is elicited. When the imposed angular frequency is equal to the inverse of the relaxation time, i.e. when $\mathrm{De}=1$ the stress maximum occurs when a strain of $\gamma_{0}\left(1+\frac{1}{\sqrt{2}}\right)$ has been acquired since the previous reversal, which occurs at an instantaneous shear rate of $\frac{\dot{\gamma}_{0}}{\sqrt{2}}$. For a general linear viscoelastic 
material, it is expected that the stress maximum will always occur when an amount of strain between $\gamma_{0}$ and $2 \gamma_{0}$ has been acquired since the previous reversal. It is equivalent to say that the stress maximum of a general linear viscoelastic material always occurs in the first quadrant of an elastic Lissajous figure. See for example Fig. 1.

\section{Methodology: experimental}

The t-SANS and stress data were acquired simultaneously by mounting an Anton Paar MCR 501 rheometer, used in the strain-controlled mode with a quartz Couette geometry, in the SANS-1 neutron beam at the SINQ spallation source at the Paul Scherrer Institute (PSI) in Villigen, Switzerland. The $\mathrm{CPyCl}-$ $\mathrm{NaSal}$ micellar solution of $6 \mathrm{wt} \%$ in $0.5 \mathrm{M} \mathrm{NaCl}$ brine in $\mathrm{D}_{2} \mathrm{O}$ (all materials from Sigma-Aldrich) was prepared one week prior to loading. We display in Fig. 2 the angle-averaged scatter curve of the sample. All measurements were performed at $28.5^{\circ} \mathrm{C}$.

We use the scalar order parameter $\left\langle P_{2}\right\rangle=\int_{0}^{\pi} f(\theta) P_{2}(\theta) \sin (\theta) \mathrm{d} \theta$ as a measure of the degree of orientation of the Kuhn segments, where $\theta$ is defined as the angle between the Kuhn segments and the flow direction in the flow-vorticity plane. ${ }^{23}$ In this relationship, $f(\theta)$ is the projection of the normalized orientation distribution function into the flow-vorticity (1 $\overline{3})$ plane, and $\theta$ is the angle between the Kuhn segment and the flow direction in this plane. The region where $I(q) \sim q^{-1}$ is, however, at quite high $q$-values. In order to find a compromise between intensity and probing the length scale of the Kuhn segments, we scanned the scalar order parameter over a range of $q$-values under stationary flow conditions using a detector distance of $6 \mathrm{~m}$ and thermal neutrons of wavelength $1.3 \pm 0.1 \mathrm{~nm}$. A small increase of $\left\langle P_{2}\right\rangle$ with increasing $q$ was observed, which saturated around the $q$-range indicated with the solid lines in Fig. 2 . This is the $q$-range used in all our analysis.

A stroboscopic SANS data acquisition scheme was employed, with an electronic trigger supplied by the rheometer to initiate the collection of data from the scattered neutrons. This technique produces up to $n=400 \mathrm{SANS}$ patterns of time resolution $\Delta t=$ $(2 \pi) /(n \omega)-1$, where $\omega / 2 \pi$ is the frequency of the applied excitation field. The trigger signals were sent at the point of maximum shear rate/zero global strain. Data were acquired over an interval on the order of one hour per experiment. We
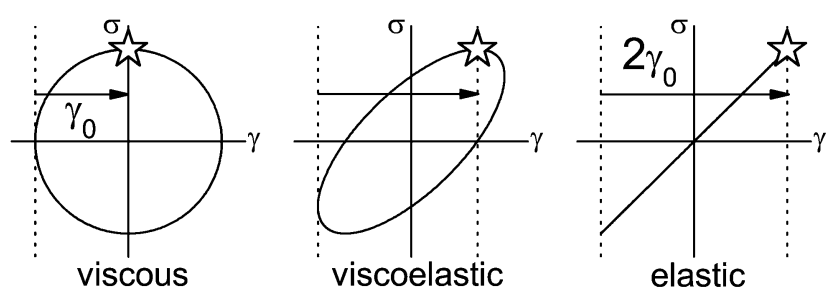

Fig. 1 Elastic Lissajous-Bowditch representations (stress plotted against strain) of three ideal linear material responses. The stress maximum, as denoted by the stars, is achieved at the point where the material has acquired $\gamma_{0}$ strain since the previous reversal in an ideal viscous material (left). For an ideal elastic solid (right), the point of greatest stress occurs at $2 \gamma_{0}$ after the previous strain reversal. A general linear viscoelastic response (center) has the stress maximum somewhere intermediate to these limits.

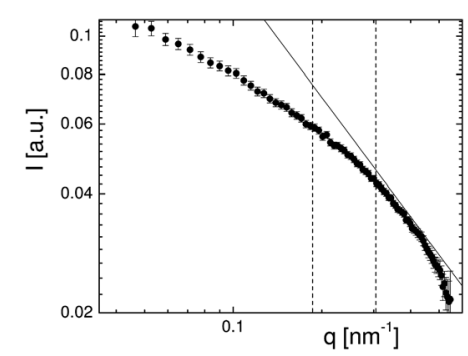

Fig. 2 Angle-averaged SANS curve of the quiescent $6 \% \mathrm{CPyCl}$. The full line indicates the $q$-range where a $q^{-1}$ dependence is found, typical for Kuhn-segments. The dashed lines mark the $q$-range from which our data are collected.

therefore investigate the steady-state oscillatory response. For each time step $\Delta t$ we measure a scatter pattern from which we derive the $\left\langle P_{2}\right\rangle$ response to the applied forces. Examples of two scattering patterns are presented in Fig. 3 .

Strain amplitudes between $\gamma \approx 0.5$ and 770 strain units, and frequencies between $\omega \approx 0.024$ and $35 \mathrm{rad} \mathrm{s}^{-1}$, which translate to Deborah numbers between 0.0073 and 10, were applied. While the Anton Paar MCR 501 is inherently a stress-controlled rheometer operating in strain-controlled mode, the rheological measurements presented here have been replicated to within experimental uncertainties using a strain-controlled ARES G2 rheometer.

\section{Results: low shear-rate amplitude regime}

The results presented in this paper, as in the theoretical work of Zhou and co-workers, ${ }^{40}$ are naturally classified as falling into one

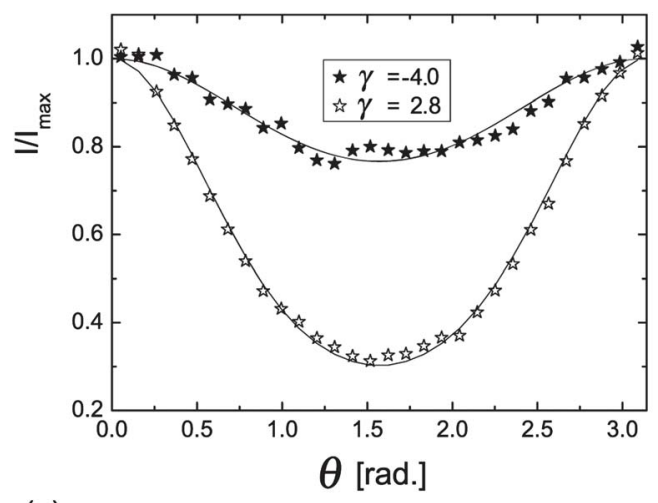

(a)
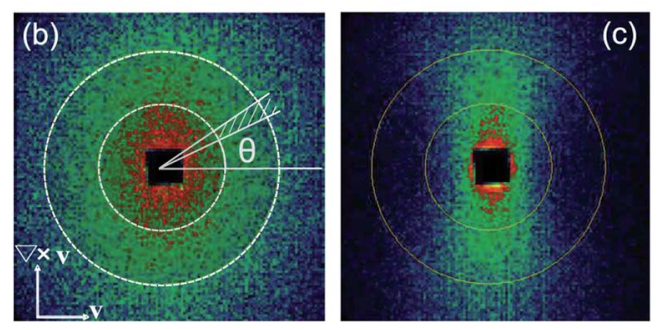

Fig. 3 The azimuthal intensity profile (a) for the two scattering patterns of the flow-velocity plane taken at a strain of $\gamma=-4.0$ (b) and $\gamma=2.7$ (c) taken during an oscillation with a frequency of $0.5 \mathrm{~Hz}(\mathrm{De} \approx 0.95)$ and a shear rate amplitude of $18.8 \mathrm{~s}^{-1}\left(\mathrm{Wi}_{0}=5.7\right)$. 
of two shear rate regimes, determined by whether the Weissenberg number amplitude is less than or greater than unity. The responses to oscillations classified by $\mathrm{Wi}_{0}>1$ will be the main focus of this work and will be investigated in subsequent sections. In this initial section we briefly investigate the case where the Weissenberg number amplitude is less than or on the order of one. In the steady-shear limit under these conditions, the flow curve is linear and proportional to $G_{0}$. Under oscillatory shear, when $\mathrm{Wi}_{0} \leq 1$, linear viscoelastic responses are elicited. This is exhibited in our analysis scheme by the amount of strain acquired since the previous reversal falling between the expected $\gamma_{0}$ and $2 \gamma_{0}$ limits, and matching, nearly perfectly, the expectations of the linear viscoelastic Maxwell model, as indicated in Fig. 4.

In the next section, we present the results of investigations of oscillatory responses characterized by Weissenberg number amplitudes greater than one. We show that in this high shear rate regime responses can be classified as belonging to one of three distinct regimes according to the amount of strain acquired at the point of maximum stress since the previous reversal.

\section{Results: high shear-rate amplitude regime}

In contrast to the response at low Weissenberg number amplitudes, high shear rate amplitude oscillations can elicit nonlinear material responses. We show in Fig. 5 the response of the system to Weissenberg number amplitudes much greater than one. It is obvious that at high frequencies, where $\mathrm{De} \geq 1$, linear responses are obtained, as in the low $\mathrm{Wi}_{0}$ case. However, as the frequency is lowered below De $=1$ (equally, as the strain amplitude is increased beyond $\mathrm{Wi}_{0} / \mathrm{De}$ ), the strain acquired since the previous

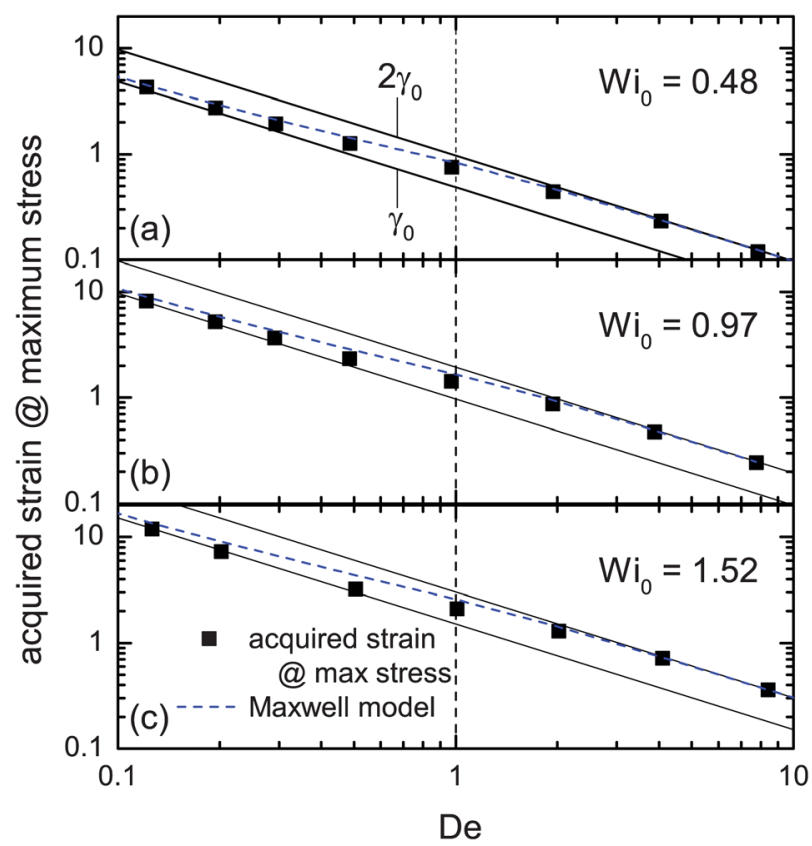

Fig. 4 The amount of strain acquired since the previous reversal at maximum stress as a function of the Deborah number for three Weissenberg number amplitudes, as indicated. In all three cases, the amount of strain acquired falls within the $\gamma_{0}$ and $2 \gamma_{0}$ limits expected for a linear viscoelastic material response. The blue dashed lines indicate the expectation values of the linear Maxwell model.
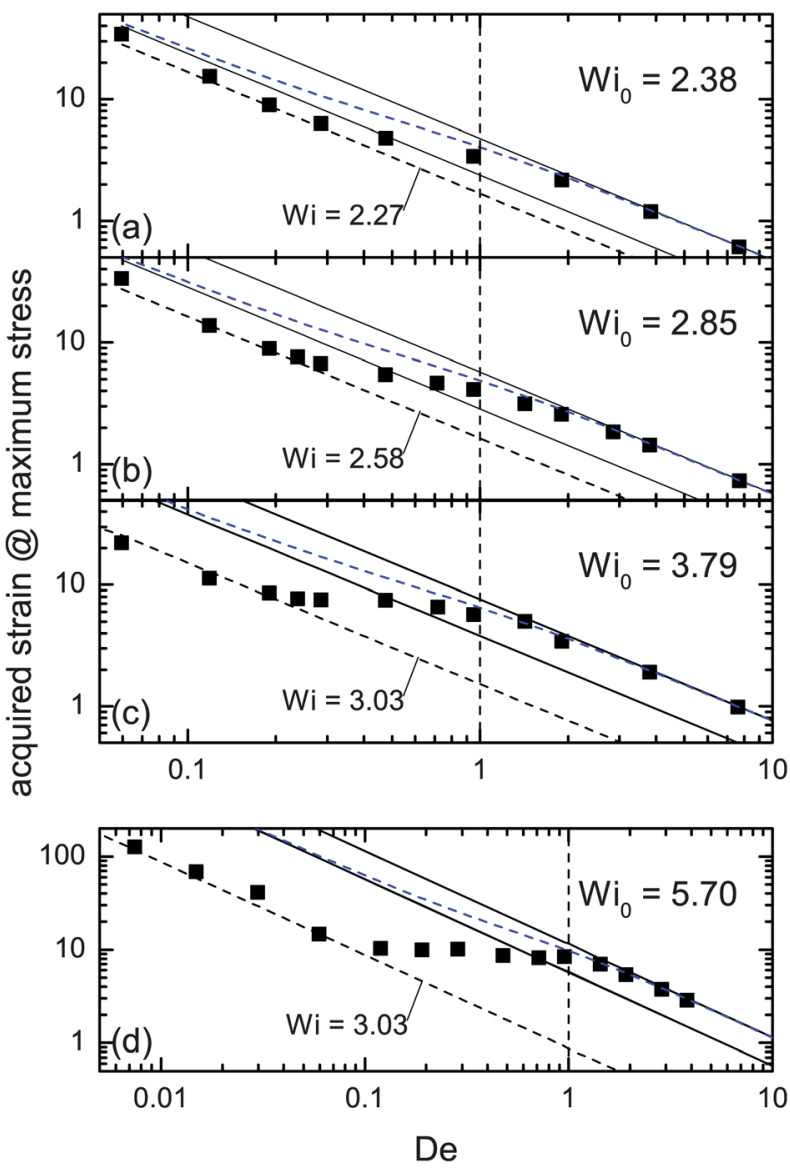

Fig. 5 The amount of strain acquired at the point of maximum stress since the previous reversal as a function of the Deborah number for Weissenberg number amplitudes greater than 1 , as indicated. At Deborah numbers $\mathrm{De} \geq 1$, in all cases, the amount of strain acquired falls within the $\gamma_{0}$ and $2 \gamma_{0}$ limits expected for a linear viscoelastic material response. At frequencies below $\mathrm{De}=1$, nonlinear responses are elicited as indicated by the experimental data falling outside the linear limits. At the very lowest frequencies, the stress maxima occur at points of constant shear rate, as indicated. The blue dashed lines indicate the expectation values of the linear Maxwell model. Symbols and lines are as in Fig. 4.

reversal at the stress maximum increasingly falls outside the linear viscoelastic limits, as indicated by the solid lines in Fig. 5. In the low-frequency limit, the maximum stress occurs at a point of constant shear rate, as indicated by the dashed angled lines in Fig. 5. As the shear rate amplitude is increased beyond $\mathrm{Wi}_{0}=1.5$, the instantaneous rate at which the stress maximum occurs in the low-De limit increases towards $\mathrm{Wi} \approx 3$. Increasing the rate amplitude beyond $\mathrm{Wi}_{0} \approx 3.79$, the response to which is indicated in Fig. 5(c), does not change this critical shear rate.

In the transition zone between the high- and low-frequency limits, the strain acquired at maximum stress occurs at a nearly constant value of strain since the previous reversal. This region starts at a Deborah number of one. We interpret the nearly constant amount of strain acquired in this transition regime as indicated by yielding dynamics. As a consequence, we refer to this regime as the 'yielding regime' and refer to the 'yield strain' as being the amount of strain acquired at the point of maximum stress at a Deborah number of one. 
In the following subsections, we will separately present and discuss the full stress and ordering responses to the three frequency regions identified above. We focus on the responses to a Weissenberg number amplitude of 5.70, an overview of which is contained in Fig. 5(d), as this dataset contains all the salient features. Where necessary, we will compare these results with those obtained at other Weissenberg number amplitudes. A particular contrast will be shown with the linear results from oscillations characterized by $\mathrm{Wi}_{0}=0.48$, an overview of which is displayed in Fig. 4(a). The low (De $\ll 1$ ) and high-frequency $(\mathrm{De}>1)$ responses are presented and discussed first, before the intermediate 'yielding regime' responses (De $\leqslant 1$ ) are interpreted in terms of interpolating between these two limits.

In each respective regime we identify linear stress-orientation relationships, where the role of intermediary parameter is played by shear rate in the low frequency regime and strain in the intermediate and high frequency regimes. The linear stressorientation relationships observed are only exhibited during time intervals shorter than a period. At high frequencies, where the strain amplitude of the excitation field is small, the Maxwell-like stress curves are decomposed to determine an elastic modulus, while the order parameter is linear with strain only above a threshold value.

\subsection{Low frequency regime}

It is expected that if the excitation frequency is low enough (De $\ll 1$ ), the oscillatory response will be equivalent to that of the steady state. We show in Fig. 6 the steady-shear steady state responses (solid lines) and the dynamic responses (symbols) from the lowest measured frequency $(\mathrm{De}=0.0075)$ for two different Weissenberg number amplitudes.

Under steady-shear conditions for values of $\mathrm{Wi} \lesssim 1$, the ordering changes exponentially with shear rate, as indicated by the dashed line in Fig. 6(a). This exponential dependence on rate could reflect a reorientation of the Kuhn segments towards the flow direction or, alternatively, a purely exponential increase in ordering, or a combination of both. Because our t-SANS detection scheme is insensitive to changes of orientation of the Kuhn segments in the flow-gradient (12) plane, we cannot make this distinction and alternative geometries need to be used. ${ }^{24}$ The resulting stress-orientation relationship, which is obtained by combining shear-rate-dependent stress and orientation curves, displays in the range $\mathrm{Wi} \lesssim 1$, a functional dependence that can be described by a quadratic dependence, as was predicted from the Giesekus model, ${ }^{24}$ see Fig. 6(f). However, for the quadratic dependence to describe the data well, a non-zero scalar term must be included, which we expect to be a function of the Deborah number which vanishes at $\mathrm{De}=0$. It should also be noted that the quadratic dependence observed previously ${ }^{24}$ also included a term that accounted for the orientation of the Kuhn segment director, which is inaccessible in our setup.

At large shear rate amplitudes and low frequencies, where De $\ll 1$, the material response is characterized by oscillations of the orientation of the Kuhn segments and the macroscopic stress about the steady-shear values as the instantaneous applied rate is increased beyond $\dot{\gamma}_{1}$ (upper arrows in Fig. 6(a) and (b), respectively). The first local maximum is found at an instantaneous shear rate of approximately $\mathrm{Wi}=3$. This value is independent of
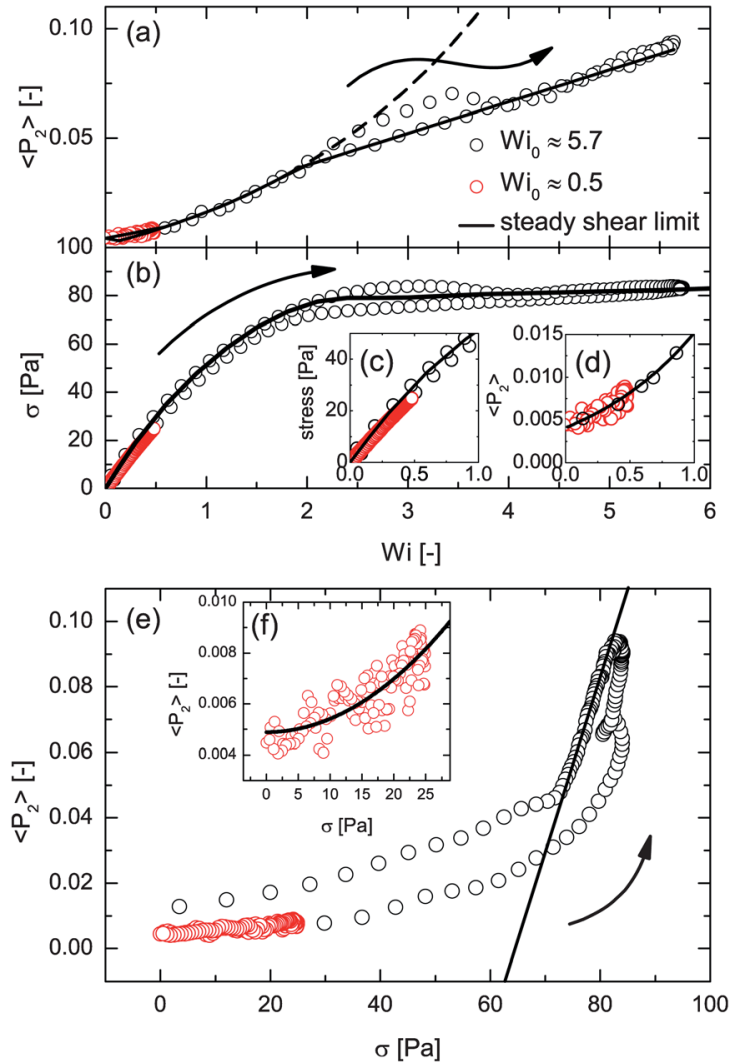

Fig. 6 Orientational order parameter (a) and stress (b) as functions of Weissenberg number (normalized shear rate) for steady-shear and oscillatory tests of $\mathrm{Wi}_{0} \approx 5.7$ (black symbols) and 0.5 (red symbols) at a normalized frequency $\mathrm{De}=0.0075$. Arrows indicate the orbital direction. Insets display close-ups of low-rate branches of stress (c) and order parameter (d). The resulting stress-orientation relationship for $\mathrm{Wi}_{0} \approx 5.7$ (e) and steady-shear (f). The solid line in (e) indicates the region of linear dependence, while in (f) the line is a fit of the form $\left\langle P_{2}\right\rangle \propto \sigma^{2}+C(\mathrm{De})$.

frequency, provided that the mechanical perturbation is applied at a frequency below some critical value $\mathrm{De}_{\mathrm{c}}$. We therefore interpret this stress/ordering feature as being the maximum of the unstable underlying flow curve. It is expected that this maximum will disappear in the limit De $\rightarrow 0$, although we have not experimentally accessed this lower limit which is indistinguishable from steady-shear.

When the instantaneous shear rate is decreasing, but still larger than the onset of the stress plateau, both the ordering of the Kuhn segments $\left\langle P_{2}\right\rangle$ and the macroscopic stress $\sigma$ change linearly with the shear rate. Hence, in this frequency regime, a linear stress-orientation relationship can be defined based on the ratios of rate dependencies of the orientation order parameter and macroscopic stress, see Fig. 6(e).

\subsection{High frequency regime}

In the high frequency case, where De $>1$, the stress can be well described by a Maxwellian sinusoid (see for instance Fig. 4 or 5), indicating a linear viscoelastic rheological response. The response to the mechanical excitation in this high-frequency regime is therefore not considered as LAOS, and linear algebraic analysis can be adopted. Despite the sinusoidal stress response, 
as exhibited in Fig. 7(b), the ordering of the Kuhn segments changes linearly with strain during an interval of the oscillation, as can be seen in the data of Fig. 7(a) where only the positive shear rate portion of the order parameter orbit (that is, the portion that is traced from $-\gamma_{0} \rightarrow \gamma_{0}$ ) is displayed to avoid confusion. The linear dependence on strain is observed only above a lower limit of the order parameter. The threshold value is approximately equal to the steady-shear value at the lower boundary of the plateau, and therefore the ordering of the Kuhn segments in the low shear-rate band. The linear dependence on strain suggests that the director of the Kuhn segments has aligned with the flow direction and that subsequent application of strain simply acts to narrow the distribution. Further, the change in order parameter with strain is independent of frequency when De $>1$, as evidenced by the coincidence of the oscillatory $\left\langle P_{2}\right\rangle$ data with the parallel lines displayed in Fig. 7(a). We therefore conclude that the Kuhn segments change their orientation with strain at a constant rate in the high frequency regime.

In order to obtain a meaningful stress-orientation relationship in this frequency regime, we divide the strain dependence of $\left\langle P_{2}\right\rangle$ by the storage modulus calculated directly from the stress orbit by

$$
G^{\prime}=G_{0} \cos (\delta)=\left(\sigma_{0} / \gamma_{0}\right) \cos \left[\sin ^{-1}\left(\gamma_{\sigma=0} / \gamma_{0}\right)\right]
$$

where $\sigma_{0}$ and $\gamma_{0}$ are the stress and strain amplitudes, and $\gamma_{\sigma=0}$ is the strain at which the instantaneous stress is zero (see Fig. 7(b)). The storage modulus increases with frequency, as is observable by the steepening of the angle between the abscissa and the major axis of the elliptical stress orbits displayed in Fig. 7(b). This can be partially related to the linear-regime tendency of the storage modulus to increase with frequency towards the plateau modulus.

In the limit of linear viscoelasticity, $G^{\prime}$ can be visualized as the slope of the decomposed $\sigma^{\prime}$ parameter, ${ }^{41}$ which is shown in Fig. 7(b) for the case where $\mathrm{De} \approx 3.81$. The so-called 'elastic

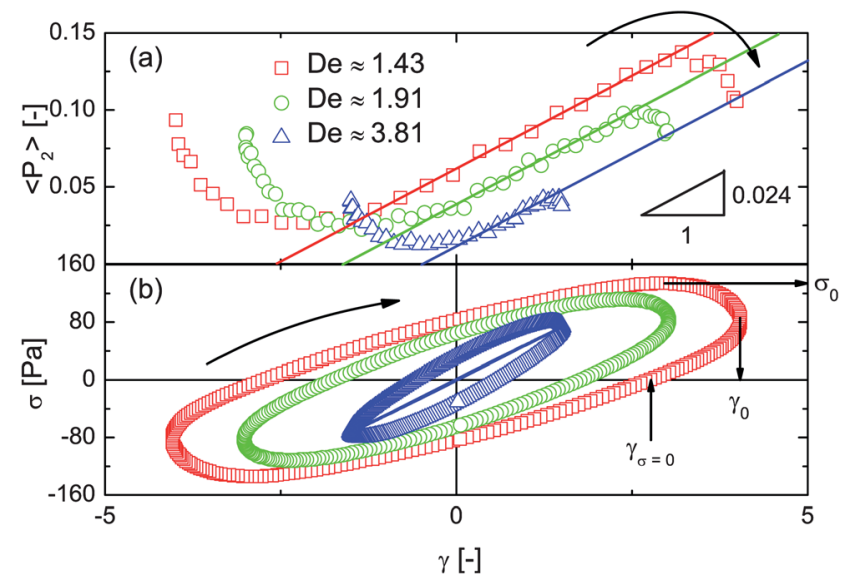

Fig. 7 Order parameter (a) and stress (b) for $\mathrm{Wi}_{0}=5.7$ and $\mathrm{De}>1$. Lines in (a) emphasize portions of order parameter orbits that change linearly with strain. The stress and strain parameters in (b) are used to calculate the storage modulus, $G^{\prime}$ (see text). The solid line is the so-called 'elastic stress' ${ }^{\prime 1}$ for the shear stress response to oscillations at $\mathrm{De} \approx 3.81$. stress' is the vertical midpoint of the elastic Lissajous figure, examples of which are displayed in Fig. 7(b).

Further, we observe a decrease in $G_{0}$ with increasing strain amplitude - we directly measure the strain-softening of the material prior to yielding. The strain-softening of the system, marked by an elastic modulus $G_{0}$ that decreases with increasing strain amplitude (decreasing frequency), is apparent from the data shown in Fig. 8(a). This behavior can be directly observed as a decrease in the slope with increasing strain amplitude of the major axis of the stress Lissajous figures in Fig. 7(b).

The time fitting parameter of the Maxwell model, $\lambda$, remains approximately constant at $0.2 \mathrm{~s}$ as shown in Fig. 8(a). At very high frequencies, the period of oscillation sets an upper limit on $\lambda$, as shown by the dotted line in Fig. 8(b). The changing modulus is not reflected in the rate at which the ordering of the system increases with strain.

The strain softening cannot be interpreted as a shear induced decrease of either the scission time or the reptation time, given that $\lambda$ does not change. Alternatively, these observations hint at the existence of a different relaxation mechanism, where branching of the micellar system plays an important role. ${ }^{42}$ It is known that similar systems to that studied here are significantly branched. ${ }^{43,44}$ In branched systems, shear flow has the effect of enforcing reorganization of branch points, which does not change relaxation times but does lead to a decrease in the modulus.

\subsection{Intermediate frequency regime}

We have examined the behavior of the WLM solution when subjected to a constant shear rate amplitude and observed at low frequencies material responses that approximate those of steadyshear tests. When subjected to high frequency oscillations, the stress response approximates that of the linear viscoelastic Maxwell model because of the combination of small strain amplitudes and high frequencies. The final results presented in this paper concern the case where large mechanical deformations are applied at intermediate frequencies, allowing us to address

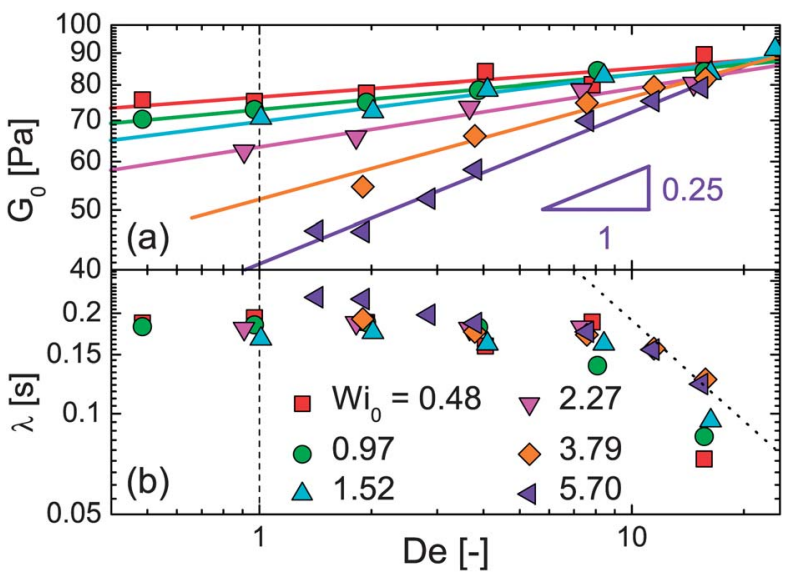

Fig. 8 The responses to all high frequency responses are well described by a single-mode Maxwell model. The fitting parameters, $G_{0}(\mathrm{a})$ and $\lambda(\mathrm{b})$, for a range of Weissenberg number amplitudes and Deborah numbers are shown. The dotted angled line in (b) represents the period of oscillation, which sets an upper limit on the time fitting parameter. 
the issue of what state the system is in before it fluidizes, as we know it must from the discussion of the low frequency regime (Fig. 6). In Fig. 9 typical responses to a Weissenberg number amplitude of $\mathrm{Wi}_{0}=5.7$, and normalized frequencies $\mathrm{De}=0.5$ to 1 are shown. As before, only the positive rate portion (from $-\gamma_{0}$ $\left.\rightarrow \gamma_{0}\right)$ of the $\left\langle P_{2}\right\rangle$ data are shown for clarity. The stress orbits (Fig. 9(b)) are clearly not elliptical, indicating that nonlinear responses are elicited from the system in this frequency/shear rate regime. While the specific shapes of the orbits may differ, there is one important similarity between all stress responses in this regime: the same maximum (yield) values of the stress and the degree of ordering are achieved at the point where approximately ten strain units since the previous flow reversal have been acquired. This amount of strain is indicated in Fig. 9(b) for one of the response orbits. The fact that we can, and do, observe macro- and microscopic properties at the moment the material yields is a major advantage of our methodology. The critical strain often corresponds approximately with the acquired strain when velocimetry measurements indicate the onset of shear banding in steady-shear step-rate tests..$^{7,17,18,20}$

Immediately prior to the point of maximum stress, the system responds nearly purely elastically as evidenced by the linearity of
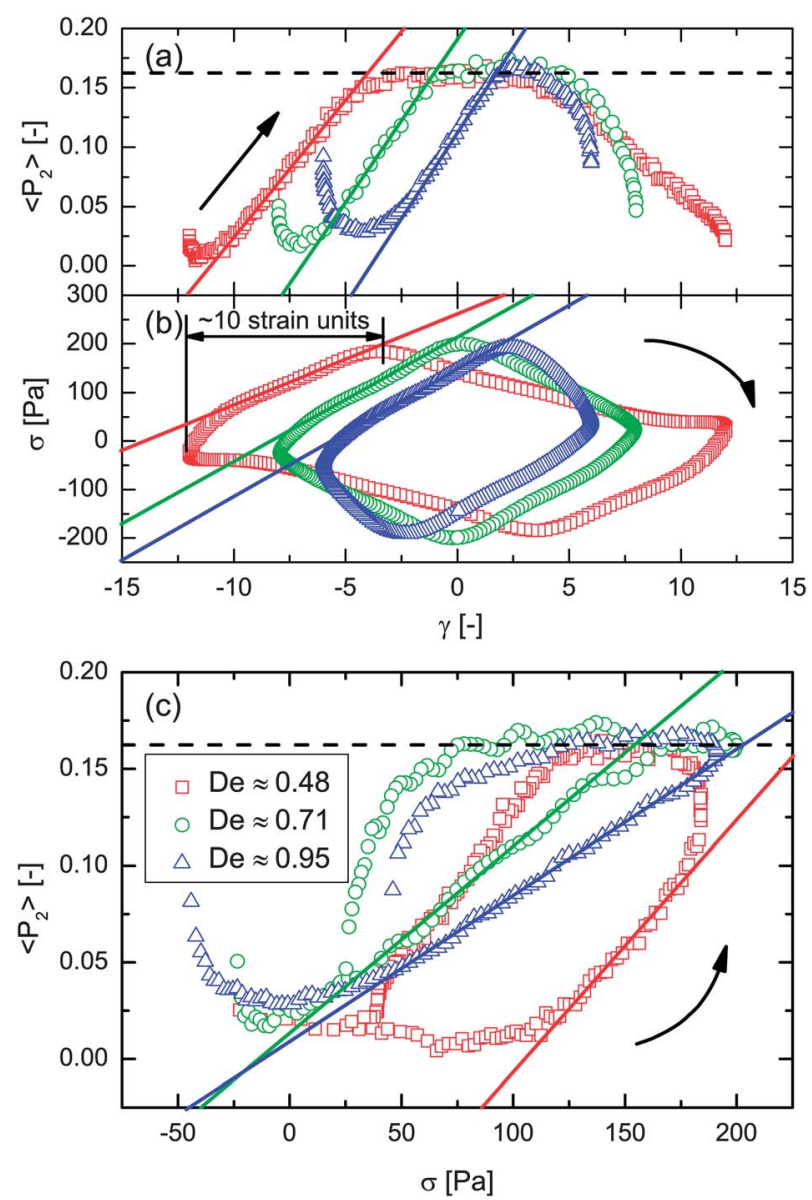

Fig. 9 Order parameter (a) and stress (b) for $\mathrm{Wi}_{0}=5.7$ and $\mathrm{De} \approx 0.5$ to 1. Lines emphasize portions of both order parameter and stress orbits that change linearly with strain. (c) The resulting stress-orientation relationship, where the solid lines indicate the portions where the relationships are linear. the stress response with strain (lines in Fig. 9(b)). We define another strain-based stress-orientation rule, see Fig. 9(c), since $\left\langle P_{2}\right\rangle$ is also linear in strain in the same strain/time window (lines in Fig. 9(a)). Following these (local and global) maxima, which we interpret as reflecting static yielding, the response becomes fluid-like (data now shown). Interestingly, $\left\langle P_{2}\right\rangle$ does not exceed the value of 0.16 , which is, as can be seen from the data of Fig. 6(a), about a factor of two greater than the ordering at the corresponding Weissenberg number (amplitude) under steadyshear, where the material is completely fluidized. The value of $\left\langle P_{2}\right\rangle=0.16$ is also more than a factor of four greater than the ordering in the low-rate band. We equate this value of $\left\langle P_{2}\right\rangle=$ 0.16 with the degree of ordering of the Kuhn segments at the point of static yielding, the point that the system starts to fluidize and form shear bands. The system can therefore be said to be 'over-oriented' at the point of yielding, compared with the steady-sheared fluid state. Prior to yielding, the material does not behave as a fluid, and therefore does not display long time transients. ${ }^{10-12}$ The persistence of the higher-ordered state $(\mathrm{De}=$ 0.48 and 0.71 in Fig. 9(a)) is interpreted as reflecting a transition in the structure of the material, from an ordered entangled state (with stress increasing) to an ordered disentangled state (with stress relaxing). Such a transition can be easily envisioned for branched systems, since it merely requires a reorganization and eventual vanishing of branch points. ${ }^{42}$

\section{Discussion}

\subsection{In situ Pipkin diagram}

We summarize the findings of this investigation by way of a Pipkin diagram, displayed in Fig. 10(a). Open symbols represent the upper and lower boundaries of the intermediate 'yielding' regime (Section 5.3). At frequencies higher than the upper boundary of this region (Section 5.2), responses are accurately described by the Maxwell model with the inclusion of softening (lower moduli recorded at larger strain amplitudes/lower frequencies). At frequencies lower than the lower boundary of the 'yielding' regime indicated by the open stars in Fig. 10(a), the stress maximum occurs at the point where an instantaneous shear rate corresponding to $\mathrm{Wi}_{\mathrm{c}}=3.03$ is applied. This apparent critical shear rate is a constant for all Weissenberg number amplitudes larger than 3.03 (see Fig. 5). At applied shear rate amplitudes lower than this value, the critical Weissenberg number is less than 3.03. We indicate this tendency in Fig. 10(a) with decreasing symbol sizes as the Weissenberg number amplitude is lowered below 3.03. For comparison, we display the predicted lower boundary of the shear banding regime of the VCM model as calculated by Zhou and co-workers. ${ }^{40}$ While our analysis is unable to unambiguously determine shear banding, our geometrical approach suggests some refinements be made to the conclusions drawn previously.

\subsection{Stress-orientation relationship}

One of the main goals of this paper has been to present a relationship between the stress and structural response of WLMs to deformation rates that render the system unstable in the longtime limit. In order to elucidate these responses, a focus has been placed on a Weissenberg number amplitude of 5.70, which is 

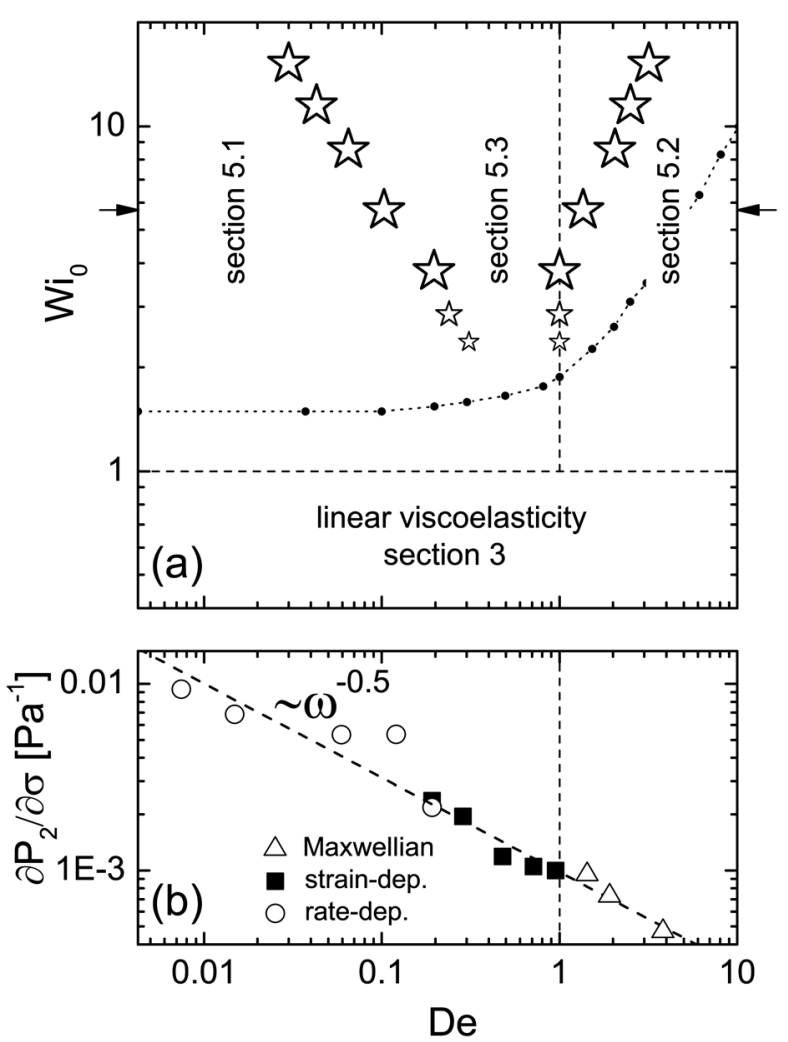

Fig. 10 (a) Pipkin diagram summarizing the findings of this investigation. (b) Stress-orientation relationship for a Weissenberg number amplitude of 5.7. Open stars in (a) mark the upper and lower boundaries of the intermediate yielding regime. The lower boundary marks the point where the yield strain coincides with a critical instantaneous shear rate. Large stars indicate $\mathrm{Wi}_{\mathrm{c}}=3.03$ while smaller stars indicate $\mathrm{Wi}_{\mathrm{c}}=2.58$ and the smallest stars $\mathrm{Wi}_{\mathrm{c}}=2.27$. The filled circles and dotted line that runs through them are the lower boundary of the shear banding regime of the VCM model as calculated by Zhou and co-workers. ${ }^{40}$ The arrows in (a) indicate the Weissenberg number amplitude of the stress-orientation rule displayed in (b). Symbols in (b) indicate data obtained from each of the three different frequency regimes.

sufficient to place the response of the material in the non-linear regime. Under such oscillatory shear rate conditions, and at all applied frequencies, we have identified time windows where both stress and the enforced ordering, $\left\langle P_{2}\right\rangle$, display a simple linear dependence on either strain (intermediate and high frequency) or shear-rate (low frequency). Under low frequency excitations there is an extended window where both $\left\langle P_{2}\right\rangle$ and stress depend linearly on the instantaneous shear rate, when the shear rate is decreasing from the maximum value, $\mathrm{Wi}_{0}=5.70$, down to the shear rate that marks the lower limit of the stress plateau (see Fig. 6). From the overlap of the macroscopic stress and microscopic ordering data with the stationary data we conclude that the system is close to its steady state response. It is known that in the steady state gradient bands are formed, where the relative sizes of the low and high shear rate bands follow the lever rule. ${ }^{6}$ In our scattering geometry, where intensity is convoluted over the gap of the Couette cell, the lever rule directly results in a linear dependence of the orientational order on the applied shear rate. This dependence has also been observed by Berret and coworkers for a similar system. ${ }^{23}$ Further, the stress displays a linear dependence on shear rate, which is due to the stress gradient that is intrinsically present in a Couette geometry.

By concatenating data obtained from the low-frequency shear rate dependence, and the intermediate and high frequency strain dependence, we obtain a stress-orientational rule, displayed in Fig. 10(b), that covers all experimental frequencies and is independent of the intermediary parameters. Interestingly, we note that this general stress-orientational rule is well described by a dependence on the inverse square of the frequency. While we know of no theoretical explanation for this dependence, we make a further note that in the high frequency case, where De $\geq 1$, the Maxwellian modulus term for the same Weissenberg number amplitude is well described by (see Fig. 8) a dependence on the frequency to the power of one quarter. We know of no reason why these parameters should be linked and suspect serendipitous values.

\section{Summary and conclusions}

We have employed a novel and robust methodology to link microstructural changes to macroscopic rheological responses in dynamic environments. While the specific results pertain directly to WLMs, we believe the methodology to be of great utility to the soft matter community in general.

In the intermediate frequency regime we have shown that prior to yielding the Kuhn segments of the WLMs 'over-align' when compared with the fluid state at the same Weissenberg number (amplitude). Further, we have shown that this 'over-alignment' is nearly independent of frequency. The fact that we can, and do, observe macro- and microscopic properties at the moment the material yields is a major advantage of our methodology. The critical/yield strain of ten corresponds approximately with the acquired strain when velocimetry measurements indicate the onset of shear banding using steady-shear step-rate tests.

Interestingly, the enforced ordering of the system, $\left\langle P_{2}\right\rangle$, does not exceed the value of 0.16 when subjected to a Weissenberg number amplitude of $\sim 5.7$. This value, as can be seen from the data of Fig. 6(a), is approximately a factor of two greater than the ordering at the corresponding Weissenberg number (amplitude) under steady-shear, where the material is completely fluidized. The value of $\left\langle P_{2}\right\rangle=0.16$ is also more than a factor of four greater than the ordering in the low-rate band. We equate the value of $\left\langle P_{2}\langle=0.16\right.$ with the degree of ordering of the Kuhn segments at the point of static yielding, the point that the system starts to fluidize and form shear bands. The system can therefore be said to be 'over-oriented' at the point of yielding, compared with the steady-sheared fluid state.

\section{Acknowledgements}

Financial support by the German Research Foundation within SFB TR 6, and the European Network Nanodirect (grant no. NMP4-SL-2008-213948). The authors thank P. Ballesta for fruitful discussions.

\section{References}

1 R. G. Larson, J. Polym. Sci., Part B: Polym. Phys., 1996, 34, 2267.

2 P. D. Olmsted, Perspectives on shear banding in complex fluids, Rheol. Acta, 2008, 47(3), 283-300. 
3 J. K. G. Dhont and W. J. Briels, Gradient and vorticity banding, Rheol. Acta, 2008, 47(3), 257-281.

$4 \mathrm{~S}$. Lerouge and J.-F. Berret, Shear-induced transitions and instabilities in surfactant wormlike micelles, Adv. Polym. Sci., 2010, 230, 171 .

5 M. E. Cates, Reptation of living polymers: dynamics of entangled polymers in the presence of reversible chain-scission reactions, Macromolecules, 1987, 20(9), 2289.

6 J.-B. Salmon, A. Colin and S. Manneville, Velocity profiles in shearbanding wormlike micelles, Phys. Rev. Lett., 2003, 90(22), 228303.

7 Y. T. Hu and A. Lips, Kinetics and mechanism of shear banding in an entangled micellar solution, J. Rheol., 2005, 49(5), 1001-1027.

8 M. R. López-González, W. M. Holmes, P. T. Callaghan and P. J. Photinos, Shear banding fluctuations and nematic order in wormlike micelles, Phys. Rev. Lett., 2004, 93, 268302.

9 E. Fischer and P. T. Callaghan, Is a birefringence band a shear band?, Europhys. Lett., 2000, 50, 803-809.

10 S. Lerouge, M. A. Fardin, M. Argentina, G. Grégoire and O. Cardoso, Interface dynamics in shear-banding flow of giant micelles, Soft Matter, 2008, 4, 1808-1819.

11 M. A. Fardin, D. Lopez, J. Croso, G. Grégoire, O. Cardoso, G. H. McKinley and S. Lerouge, Elastic turbulence in shear banding wormlike micelles, Phys. Rev. Lett., 2010, 104, 178303.

12 M. A. Fardin, B. Lasne, O. Cardoso, G. Grégoire, M. Argentina, J. P. Decruppe and S. Lerouge, Taylor-like vortices in shearbanding flow of giant micelles, Phys. Rev. Lett., 2009, 103, 028302.

13 M. A. Fardin, T. J. Ober, C. Gay, G. Gregoire, G. H. McKinley and S. Lerouge, Potential "ways of thinking" about the shear-banding phenomenon, Soft Matter, 2012, 8(4), 910-922.

14 M. Doi and S. F. Edwards, Dynamics of rod-like macromolecules in concentrated solution part 2, J. Chem. Soc., Faraday Trans. 2, 1978, 74(5), 918-932.

15 J. K. G. Dhont and W. J. Briels, Inhomogeneous suspensions of rigid rods in flow, J. Chem. Phys., 2003, 118(3), 1466-1478.

$16 \mathrm{H}$. Giesekus, A simple constitutive equation for polymer fluids based on the concept of deformation-dependent tensorial mobility, J. NonNewtonian Fluid Mech., 1982, 11(1-2), 69-109.

17 E. Miller and J. P. Rothstein, Transient evolution of shear-banding wormlike micellar solutions, J. Non-Newtonian Fluid Mech., 2007, 143(1), 22-37.

18 O. Radulescu, P. D. Olmsted, J. P. Decruppe, S. Lerouge, J.-F. Berret and G. Porte, Time scales in shear banding of wormlike micelles, Eur. Phys. J. E: Soft Matter Biol. Phys., 2003, 62, 230-237.

19 S. Lerouge, J. P. Decruppe and J.-F. Berret, Correlations between rheological and optical properties of a micellar solution under shear banding flow, Langmuir, 2000, 16, 6464-6474.

20 M. P. Lettinga and S. Manneville, Competition between shear banding and wall slip in wormlike micelles, Phys. Rev. Lett., 2009, 103, 248302.

21 P. Ballesta, M. P. Lettinga and S. Manneville, Superposition rheology of shear-banding wormlike micelles, J. Rheol., 2007, 51(5), 1047-1072.

22 S. Förster, M. Konrad and P. Lindner, Shear thinningand orientational ordering of wormlike micelles, Phys. Rev. Lett., 2005, 94, 017803.

23 J.-F. Berret, D. C. Roux and P. Lindner, Structure and rheology of concentrated wormlike micelles at the shear-induced isotropic-tonematic transition, Eur. Phys. J. B, 1998, 5, 67-77.

24 M. E. Helgeson, P. A. Vasquez, E. W. Kaler and N. J. Wagner, Rheology and spatially resolved structure of cetyltrimethylammonium bromide wormlike micelles through the shear banding transition, J. Rheol., 2009, 53(3), 727-756.

25 M. E. Helgeson, M. D. Reichert, Y. T. Hu and N. J. Wagner, Relating shear banding, structure, and phase behavior in wormlike micellar solutions, Soft Matter, 2009, 5, 3858-3869.
26 M. E. Liberatore, F. Nettesheim, P. A. Vasquez, M. E. Helgeson, N. J. Wagner, E. W. Kaler, L. P. Cook, L. Porcar and Y. T. Hu, Microstructure and shear rheology of entangled wormlike micelles in solution, J. Rheol., 2009, 53(3), 441-458.

27 E. Cappelaere, J. F. Berret, J. P. Decruppe, R. Cressely and P. Lindner, Rheology, birefringence, and small-angle neutron scattering in a charged micellar system: evidence of a shear-induced phase transition, Phys. Rev. E: Stat. Phys., Plasmas, Fluids, Relat. Interdiscip. Top., 1997, 56(2), 1869-1878.

28 A. Raudsepp and P. T. Callaghan, A rheo-optical study of shear rate and optical anisotropy in wormlike micelles solutions, Soft Matter, 2008, 4, 784-796.

29 M. R. López-González, W. M. Holmes and P. T. Callaghan, Rheo-nmr phenomena of wormlike micelles, Soft Matter, 2006, 2, 855-869.

30 J. P. Decruppe, R. Cressely, R. Makhloufi and E. Cappelaere, Flow birefringence experiments showing a shear-banding structure in a ctab solution, Colloid Polym. Sci., 1995, 273(4), 346-351.

31 R. Makhloufi, J. P. Decruppe, A. Aitali and R. Cressely, Rheo-optical study of worm-like micelles undergoing a shear banding flow, Europhys. Lett., 1995, 32(3), 253-258.

32 T. Shikata, S. J. Dahman and D. S. Pearson, Rheo-optical behavior of wormlike micelles, Langmuir, 1994, 10, 3470-3476.

33 Ch. Münch, H. Hoffmann, J. Kalus, K. Ibel, J. C. Neubauer and U. Schmelzer, Decay of a shear-induced phase transition on a micellar solution measured by time-dependent small-angle neutron scattering, J. Appl. Crystallogr., 1991, 24, 740-746.

34 Ch. Münch, H. Hoffmann, K. Ibel, J. Kalus, J. C. Neubauer, U. Schmelzer and J. Selbach, Transient small-angle neutron scattering experiments on micellar solutions with a shear-induced structural transition, J. Phys. Chem., 1993, 97, 4514-4522.

35 B. Lonetti, J. Kohlbrecher, L. Willner, J. K. G. Dhont and M. P. Lettinga, Dynamic response of block copolymer wormlike micelles to shear flow, J. Phys.: Condens. Matter, 2008, 20, 404207.

36 S. A. Rogers and M. Paul Lettinga, A sequence of physical processes determined and quantified in LAOS: application to theoretical nonlinear models, J. Rheol., 2012, 56, 1-25.

37 K. Hyun, M. Wilhelm, C. O. Klein, K. S. Cho, J. G. Nam, K. H. Ahn, S. J. Lee, R. H. Ewoldt and G. H. McKinley, A review of nonlinear oscillatory shear tests: analysis and application of large amplitude oscillatory shear (LAOS), Prog. Polym. Sci., 2011, 36(12), 1697-1753.

38 S. A. Rogers, B. M. Erwin, D. Vlassopoulos and M. Cloitre, A sequence of physical processes determined and quantified in LAOS: application to a yield stress fluid, J. Rheol., 2011, 55, 435-459.

39 A. J. Giacomin, R. B. Bird, L. M. Johnson and A. W. Mix, Largeamplitude oscillatory shear flow from the corotational Maxwell model, J. Non-Newtonian Fluid Mech., 2011, 166(19-20), 10811099.

40 L. Zhou, L. P. Cook and G. H. McKinley, Probing shear-banding transitions of a model entangled wormlike micellar solution using large amplitude oscillatory shearing (LAOS) deformations, J. NonNewtonian Fluid Mech., 2010, 165, 1462-1472.

41 K. S. Cho, K. Hyun, K. H. Ahn and S. J. Lee, A geometrical interpretation of large amplitude oscillatory shear response, J. Rheol., 2005, 49(3), 747-758.

42 W. J. Briels, P. Mulder and W. K. den Otter, Simulations of elementary processes in entangled wormlike micelles under tension: a kinetic pathway to y-junctions and shear induced structures, J. Phys.: Condens. Matter, 2004, 16(38), S3965-S3974.

43 U. Olsson, J. Börjesson, R. Angelico, A. Ceglie and G. Palazzo, Slow dynamics of wormlike micelles, Soft Matter, 2010, 6, 1769-1777.

44 M. Monduzzi, U. Olsson and O. Söderman, Bicontinuous micellar solutions, Langmuir, 1993, 9, 2914-2920. 